\title{
Hydrogen gas sensor using double SAW resonator system
}

\begin{abstract}
This paper presents a hydrogen gas sensor based on Double Surface Acoustic Wave Resonator (DSAWR) system configuration. Two commercial SAW resonators were employed to develop the DSAWR system. The sensing layer was prepared using functionalized Carbon Nanotubes (CNT) with polyaniline nanofibers. The sensing layer was integrated into the DSAWR system and measurements were carried out for hydrogen gas between $1 \%$ to $2 \%$ concentrations. Results obtained showed response of the sensor to hydrogen gas with a minimum detection limit of $1 \%$ and good response and recovery time.
\end{abstract}

Keyword: Hydrogen gas sensor; DSAWR system; Gas detectors; Sensing layers 